\title{
COUPLING MODELS OF ROAD TUNNEL TRAFFIC, VENTILATION AND EVACUATION
}

\author{
Blaž LUIN ${ }^{\star}$, Stojan PETELIN \\ Faculty of Maritime Studies and Transport, University of Ljubljana, Slovenia \\ Received 2 October 2018; revised 31 July 2019; accepted 6 November 2019; \\ first published online 20 February 2020
}

\begin{abstract}
As road tunnel accidents can result in numerous fatalities and injuries, attention must be paid to accident prevention and management. To address this issue, use of integrated tunnel model for system evaluation and training of road tunnel operators on computer simulator is presented. A unified tunnel model, including traffic, meteorological conditions, ventilation and evacuation that is presented. An overview of simulation models, simulator architecture and challenges during the development are discussed. The integrated tunnel model is used as a core of a simulation system that is capable of reproducing tunnel accidents in real time and it interfaces with Supervisory Control And Data Acquisition (SCADA) interfaces used in real tunnel control centres. It enables operators to acquire experience they could otherwise get only during major accidents or costly exercises. It also provides the possibility for evaluation of tunnel control algorithms and Human Machine Interfaces (HMIs) for efficient operation of all safety systems during upgrades and maintenance. Finally, application of the model for accident analysis and optimization of emergency ventilation control is presented where it was used to identify cause of emergency ventilation malfunction and design fault.
\end{abstract}

Keywords: road tunnel, simulation, safety, ventilation, traffic, tunnel fire, emergency, visualization, operator training, incident management.

\section{Introduction}

Although there are plenty of efforts to increase the level of safety, the tunnels remain areas of increased risk as they are semi-enclosed spaces where explosions, fires and smoke can have more devastating effects than in the open spaces. There is a great deal of focus on tunnel safety after catastrophic road tunnel accidents that have occurred in the past, such as the Mont Blanc, Tauern and Gotthard tunnel fires whose causes were widely analysed and described (Voeltzel, Dix 2004). The Mont Blanc fire in 1999 resulted in 39 fatalities, the Tauern tunnel fire in the same year resulted in 12 fatalities and 42 injured and 60 vehicles collided during the accident. As a result, the EU issued a Directive 2004/54/EC on tunnel safety to prescribe minimum safety requirements for road tunnels that requires mandatory risk assessment for all road tunnels on the trans-European road network that are longer than $500 \mathrm{~m}$ (EC 2004). A reminder that tunnel safety remains an interesting topic is the Yanhou tunnel fire (China) that occurred in 2014 and resulted in 40 fatalities.
As prescribed by the directive, several risk assessment methodologies evolved. The most widely used is the World Road Association (PIARC) and Organisation for Economic Co-operation and Development (OECD) Quantitative Risk Assessment Model (QRAM) (Cassini et al. 2003; Cassini 1998). It assumes many different scenarios of dangerous and non-dangerous goods accidents. When evaluation of risk minimization measures that were not included in the methodology were needed, extensions to the methodology were developed (Petelin et al. 2010). Results from tunnel risk analyses are key data for implementation of risk mitigation measures, which may be also be chosen by consulting expert opinions and multi-criteria analyses (Hashemkhani Zolfani et al. 2013). The results obtained with the above-mentioned methodologies often pointed out importance of prompt accident detection and response in order to minimize consequences. The tunnel operator response in case of emergency is crucial as it is the first who should take actions including informing and

${ }^{*}$ Corresponding author. E-mail: blaz.luin@fpp.uni-lj.si 
coordinating other emergency services. For this reason, risk for tunnel users can vary greatly due to the great variability of tunnel operator responses and response times (Gandit et al. 2009).

Experience from past accidents showed that the road tunnel operators were often not sufficiently prepared because they never experienced a major emergency event. While minor incidents occur daily, major tunnel accidents such as fires and explosions occur rarely. Some operators might experience such an event once in a career, some never; therefore, lacking sufficient experience they could not handle situation in an optimum way.

To sum up, interactions between traffic and ventilation control and communication between tunnel users and emergency services can be complex and demanding to understand. Improper interaction can increase damage and the number of fatalities (Carvel et al. 2009). For example, if air velocity is too high, more oxygen might help a fire spread faster or in the wrong direction - air might push smoke into tunnel users and emergency services. One way to approach complexity of tunnel control is through proposed automated decision-making system (Alvear et al. 2013).

The problem of efficient tunnel control could also be approached in a similar way as in the aerospace, chemical and nuclear industries - by training on computer simulators. These industries have a long history of standardized equipment testing prior to the commissioning and personnel training according to the standards IEC TS 62603-1:2014 and IEC 62381:2012. In the standard IEC TS 62603-1:2014, Factory Acceptance Tests (FAT) and Site Acceptance Tests (SAT) it is specified what evaluations must be carried out on Human-Machine Interfaces (HMIs), controllers, terminations, fields and process definitions; provisions for life cycle support, including personnel training are specified, also, a demo unit of a system at the site is suggested.

The aim of this work is to develop a unified model of a road tunnel traffic, ventilation and evacuation. It can serve as a core of a computer-based simulator, which includes traffic, ventilation and evacuation sub-models that are computed in real time, representing a demo unit for a road tunnel. It could not only be applied for operator training, but also for evaluation of different accident scenarios during the tunnel design and risk analyses. Therefore, an overview of state-of-the-art models that were considered to be incorporated into the simulator will follow in the next sections.

\section{Approaches to tunnel traffic, ventilation and evacuation modelling}

\subsection{Road traffic microsimulation}

Microscopic models are detailed models that simulate individual vehicles. Since the simulator requires implementation of visualization, a lower level of detail than microscopic is not sufficient. The microscopic models are comprised of vehicle following and lane-changing models, except in some rare cases where 2D simulation is assumed. Among the most popular are the Fritzsche (1994), Wiedemann (1974) and IDM (Intelligent Driver Model) - Kesting et al. (2007, 2010), Treiber (2013), Gipps (1981) models. It is common among them that speed, acceleration and longitudinal position are continuous variables and lateral position (lane) is a discrete variable. The Fritzsche (1994) and Wiedemann (1974) approaches to modelling represent real driver actions more realistically as they take into consideration physiological limitation of human drivers, especially in terms of ability to sense and react to changes in speed.

Most of the traffic microsimulation software uses a particular vehicle following model that cannot be changed or mixed with different models; for example, VISSIM (http://vision-traffic.ptvgroup.com) uses the Wiedemann model, paramics uses Fritzsche and AIMSUN (http://www.aimsun.com) uses a modification of the Gipps model (Wiedemann 1974; Fritzsche 1994; Gipps 1981). For research purposes Simulation of Urban MObility (SUMO) tool is becoming increasingly popular as it is open source and allows modifications to be made to any part of the code (Krajzewicz et al. 2012). Even without modifications of the code, it provides many configuration options not usually found in other packages such as choice of a vehicle following, lane changing and emissions models and mixing of them in the same simulation scenario.

It is common among all these models that lane is a discrete variable and longitudinal position is a continuous variable. This causes some difficulties during visualization where smooth movements are required for realistic representation. Similar issues are found when using such models in driving simulators, which often use 3D virtual reality interfacing in order to provide realistic visualization for evaluation of driver responses (Wan et al. 2018). Real-time and faster computation is easily achieved using the traffic microsimulation models, where the road network is a single road tunnel with surrounding road sections.

\subsection{Tunnel ventilation models}

There are three basic approaches to simulating tunnel ventilation: 1D, zone and Computational Fluid Dynamics (CFD) models. 1D models are comprised of continuity, momentum and energy conservation equations. Simplified versions assume stationary conditions and use variations of the Bernoulli equation for flow estimation. A notable model of this kind is the IDA Tunnel Ventilation Model (Sahlin 1996; Sahlin, Grozman 2003). Zonal models assume zones of constant temperature and gas concentrations. Examples of those models are Consolidated model of Fire growth And Smoke Transport (CFAST) and Pressurized zOnal Model with Air-diffuser (POMA) (Haghighat et al. 2001; Peacock et al. 2013). The zonal models are semi-2D models that consider simplified species transfer equations between the zones. Such approach would appear complicated in case of constantly changing boundary conditions due to moving vehicles. 
Since road tunnels are comprised of a large area, detailed simulation would be computationally demanding. Therefore, by sacrificing a certain degree of detail, simplified models such as multi-zone emerged (Suzuki et al. 2003). An advantage of the simplified models is that they take into consideration effects that are specific to the tunnels such as the piston effect that is caused by the vehicles pushing air in the driving direction. The 3D CFD simulations of vehicle fires in bidirectional tunnels yield significant impact of moving vehicles on fire dynamics (Caliendo et al. 2012a, 2012b). The increases of temperature around the fire was observed in the range of $12 \ldots 29 \%$. Apart from the air flow, tunnel wall temperature has a major effect on air temperature inside the tunnel and wall temperature conduction and radiation models are part of the $1 \mathrm{D}$ and multi-layer zone models as well.

There have also been attempts to simulate fires with virtual reality techniques, that were not specifically targeted at road tunnels and the focus was not on real-time simulations ( $\mathrm{Xu}$ et al. 2014). Instead, physical simulation of the fire and air movement was done in advance and the results were used for realistic visualization. The same approach was also applied to the road tunnels, where fire scenarios were simulated in advance with the Fire Dynamics Simulator (FDS) and visualized using the 3D computer graphics (Cha et al. 2012). Such approach works on scenarios simulated and solved in advance, but does not show immediate impact of operator actions on the situation inside the tunnel.

The tunnel simulation models serve as a basis for design and evaluation of tunnel ventilation algorithms, which can be a demanding task even in the case of simple longitudinally ventilated tunnels when appropriate pressure difference at the tunnel cross passages must be maintained. Too low pressure difference will cause smoke to enter the evacuation tube and too high pressure difference will require application of excessive force to open the cross passage door (Luin et al. 2011).

\subsection{Evacuation models}

Evacuation models can be divided into continuity, Cell Automata (CA) and multi-agent models. The continuity models are based on flow equations. CA models assume a mesh of cells that represents possible discrete positions of humans. Cell sizes are usually the size of a standing man and step sizes can be a cell or two, depending on walking speed. Multi agent models usually assume continuous position variables and each evacuating person is moving by the predefined rules or even artificial intelligence (Shi et al. 2009). Agents can have memory to remember and learn from past evacuation attempts.

Several applications of tunnel evacuations modelling have been carried out (Caliendo et al. 2012a, 2012b; Capote et al. 2013). The most obvious problem for tunnel evacuation scenarios is that accident position and vehicle distribution is always different in each event. Therefore, initial conditions should be obtained from the traffic and ventilation models. The simulations were carried out using different cell evacuation models with $0.5 \mathrm{~m}$ wide square cells and the results were consistent among all the models. Therefore, if evacuation model is sufficiently tuned, the results should be realistic given the initial conditions are appropriately defined.

An advantage of cell models is also that they are computationally undemanding and are suitable for simulations much faster than real-time, which means many simulation runs can be evaluated for risk assessment and optimization purposes.

\section{Integrated tunnel model}

In real tunnels communication system consist of a physical layer and networking protocols that are used to connect controllers and cameras. In modern tunnels equipment is usually controlled through a ring fibre optic ethernet connection to ensure redundancy. The data is fed to the server-side application; in our example, a data forwarder or a central controller stores states of the entire tunnel equipment by communicating with tunnel equipment Programmable Logic Controllers (PLCs) and supplies the data to subscribers such as Supervisory Control And Data Acquisition (SCADA) user interface, remote monitoring systems, data log recorders, etc. The tunnel PLCs are used to control all the tunnel equipment, such as ventilation fans, lighting, traffic signalization (Variable Message Signs - VMS, traffic lights) and environmental sensors (visibility, air quality). It also stores data history in the Structured Query Language (SQL) database that can be used for analysis of equipment malfunction or incidents. In Figure 1, an overview of the system is shown.

In the simulator, the tunnel is replaced by a computer model that behaves as a virtual tunnel, capable of simulating physical phenomena, sensors and PLCs. The components are also connected using Ethernet connection and TCP/IP protocol. As opposed to the real tunnels, where sensors are connected directly to controllers that interface them to a data forwarder through TCP/IP protocol, in the simulator a communication module replaces the PLCs and communicates with the data forwarder application (Figure 2).

Key simulator components are the user interface, the communications system and the simulator core. The overview of the architecture is shown in Figure 2. The HMI is comprised of operator and instructor user interfaces. Operator user interface is an exact replication of a control room workplace with all the screens that are used by the operators: video surveillance system, SCADA display, video detection interface, dangerous goods vehicle detection system, etc. The SCADA system did not need any modifications, as opposed to the video detection and surveillance systems that had to be replaced by simulated modules. The video processors are not needed in the simulator since the data can be obtained directly from the simulation core. 


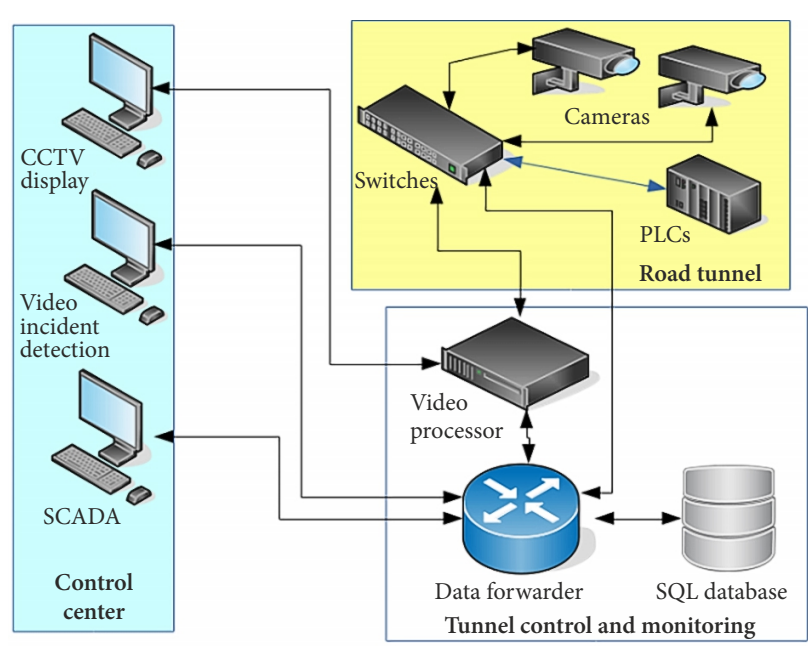

Figure 1. Tunnel control system

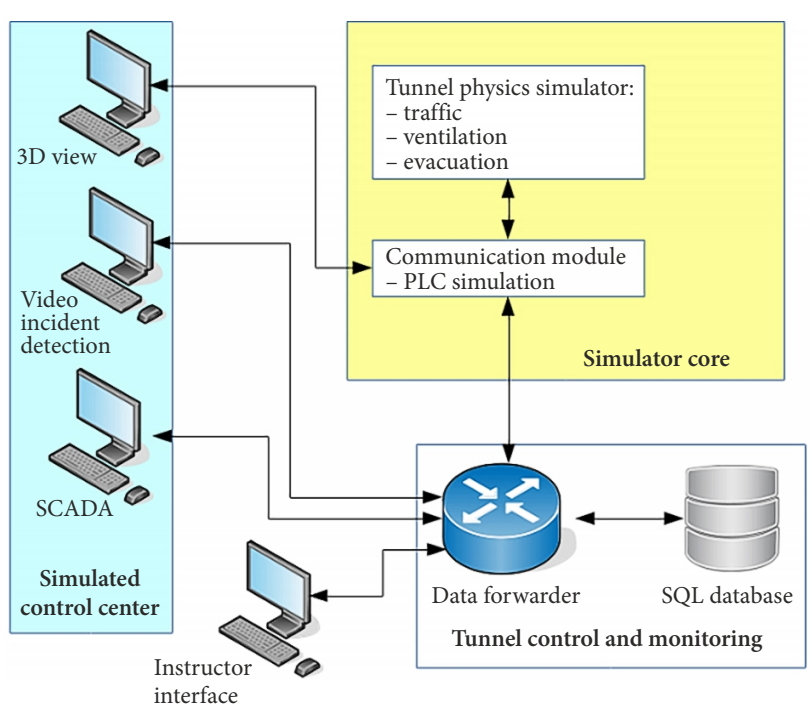

Figure 2. Simulator architecture

\subsection{Simulator core models}

The simulator core is the most vital part of simulator. It is comprised of physical and other phenomena models that occur in a tunnel during simulated training sessions. These include models of all tunnel equipment. Among phenomena that are simulated by the simulator core are traffic, ventilation and meteorological conditions models and an evacuation model.

The core models have been implemented in $\mathrm{C}++$ programming language for performance reasons, but simulation state exports may be exported in MATLAB (https://www.mathworks.com), SQL database or comma separated value file format. Core models are implemented in different parallel threads that require synchronization of the data.

Data flow of boundary conditions can be seen in the Figure 3. Interconnected models mean that the state of one model is the boundary condition of the other one and vice versa. For example, the ventilation model includes the

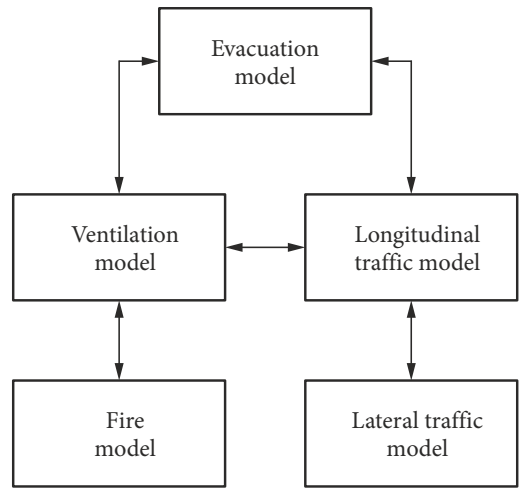

Figure 3. Data flow between the models

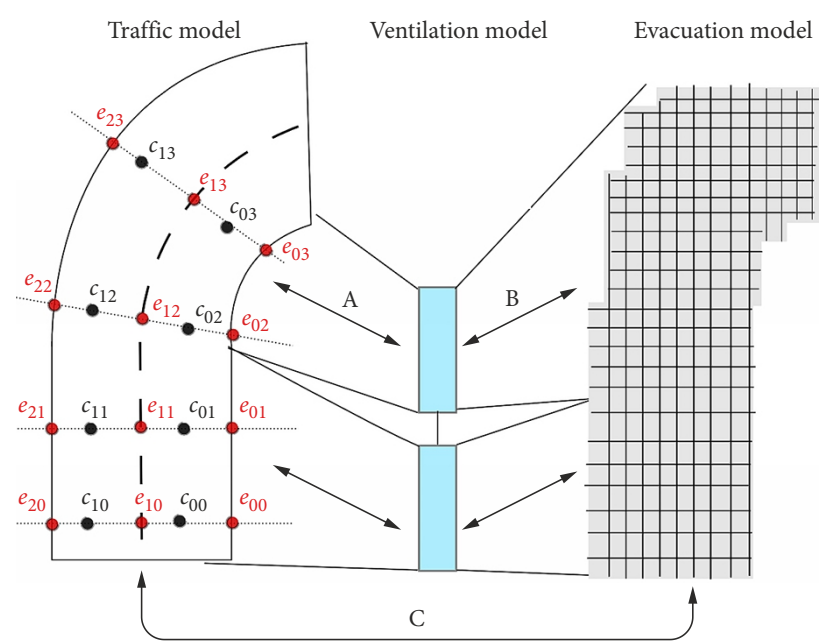

Figure 4. Coordinate system mapping between different simulator components

fire model, which may limit visibility and driveability conditions. On the other hand, moving vehicles in the traffic model push air in the driving direction, causing the piston effect and influence longitudinal air flows through the tunnel.

In Figure 4, mappings between different coordinate systems in each of the models are illustrated. The ventilation model is represented as a topology of pipes inside, which simulation is carried out in $1 \mathrm{D}$, the traffic model is represented as a road network topology, but for visualization purposes vehicle positions are assigned between as a set of 3D arrays of points for each lane. The evacuation model is a $2 \mathrm{D}$ mesh of cells. Therefore 3 transformations had to be defined:

- A - between traffic and ventilation model;

- B - between ventilation and evacuation model;

- C - between evacuation and traffic model.

Different coordinate systems of the models also include differently split sections. For example, single traffic section may be divided into two different ventilation sections as it is shown in the Figure 4 . This is the consequence of different tunnel cross-sections. For example, a three-lane traffic section may be in circular and perpendicular tunnel tube or even a section with a cross-passage. 


\subsection{Ventilation model}

Due to real-time processing requirements, the ventilation model that was used is a $1 \mathrm{D}$ ventilation model similar to that described in research by Petelin et al. (2010), except that it was generalized to support multiple branches that represent multi-tube tunnels and cross passages. The road tunnel chosen in the model contains underground road junctions, therefore it was represented as pipe branches and junctions shown in Figure 5.

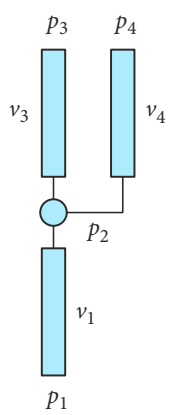

Figure 5. Ventilation model sections

The pressures $p_{1}$ to $p_{4}$ at the junctions 1 to 4 and air velocities $v_{1}, v_{3}$ and $v_{4}$ in branches 1,3 and 4 are connected using the equations:

$$
\begin{aligned}
& p_{1}-p_{2}=\Delta p_{\text {piston }, 1}+\Delta p_{\text {fans }, 1}+\Delta p_{\text {meteo }, 1}+\Delta p_{\text {fric }, 1} \\
& p_{2}-p_{3}=\Delta p_{\text {piston }, 3}+\Delta p_{f a n s, 3}+\Delta p_{\text {meteo }, 3}+\Delta p_{\text {fric }, 3} \\
& p_{2}-p_{3}=\Delta p_{\text {piston }, 3}+\Delta p_{f a n s, 3}+\Delta p_{\text {meteo }, 3}+\Delta p_{\text {fric }, 3}
\end{aligned}
$$

where: $\Delta p_{\text {piston }}$ is piston effect caused by the vehicles; $\Delta p_{\text {fans }}$ is pressure increase due to fans; $\Delta p_{\text {meteo }}$ is impact of meteorological conditions; $\Delta p_{\text {fric }}$ is friction on the tunnel walls.

When air velocities are calculated from the pressure equations, the species movement (combustion products, exhaust gases, smoke) are calculated using cells of a 1D grid. The pressure drops $\Delta p_{\text {piston }}, \Delta p_{\text {fans }}, \Delta p_{\text {meteo }}$ and $\Delta p_{\text {fric }}$ are all functions of longitudinal air velocities $v_{i}$. Species concentration in $i$-th cell changes in a single time step $d t$ according to:

$$
\begin{aligned}
& c_{i}(t+d t)= \\
& \frac{c_{i}(t) \cdot V_{i} \cdot \rho_{i}(t)+c_{\text {in }}(t) \cdot V_{\text {in }} \cdot \rho_{\text {in }}(t)-c_{i}(t) \cdot V_{\text {out }} \rho_{i}(t)}{V_{i} \cdot \rho_{i}+V_{\text {in }} \cdot \rho_{\text {in }}(t)-V_{\text {out }} \cdot \rho_{\text {out }}(t)}
\end{aligned}
$$

where: $c_{i}, \rho_{i}, V_{i}$ are mass specific species concentration, density and volume of $i$-th cell; $c_{i n}, \rho_{i n}, V_{i n}$ represent concentration, density and volume of inflow from neighbouring cells; $V_{\text {out }}$ is volume represents flow out of the $i$-th cell.

The time-step during the simulation was limited by:

$$
\delta t_{\max }<\frac{l}{\max \left(v_{0}, \ldots, v_{n}\right)},
$$

where: $l$ is section length; $v_{i}$ is air velocity in $i$-th section.
The $1 \mathrm{D}$ model has the shortcoming of not being able to simulate vertical smoke movement, therefore a simplified approach was used in order to mimic smoke stratification behaviour, which is important for the tunnel operators to understand. It was achieved by simulating smoke vertical movement depending on the longitudinal air velocity.

\subsection{Traffic simulation model integration}

Since vehicle following and lane changing models are generally known and are well studied, this section will deal only with problems related to implementation of simulation in the integrated model of a tunnel related to $3 \mathrm{D}$ visualization. Most vehicle following models assume space is a continuous variable and it can therefore be visualized smoothly and continuously if at least 20 iterations/s are calculated to achieve smooth perception of the visualization.

To the contrary, a majority of lane change models consider lane a discrete variable that either changes instantly or the vehicle remains in a transition state for a certain period of time. In either case, visualization is not smooth if standard models like Fritzsche or MOBIL are used unless intermediate position interpolation is carried out (Fritzsche 1994; Kesting et al. 2007). Another solution for smooth visualization would be development of a true $2 \mathrm{D}$ traffic model, but that would make sense only if drivers often ignore lane markings in the simulated area. In Figure 6 continuous and discrete lane change is shown.

Discrete lane changes are convenient for traffic flow analysis as they are not computationally demanding. On the other hand, they don't provide all the necessary information that is needed for smooth visualization.

The model had to provide $3 \mathrm{D}$ visualization, which required interpolation from $1 \mathrm{D}$ to $3 \mathrm{D}$ space regardless of which type of lane change model was used. This implies the use of a discrete model that is less computationally demanding and very common for simulation of drivervehicle agents.

As can be seen in Figure 7:

$$
\vec{c}_{i j}=\left(c_{x, i j}, c_{y, i j}, c_{z, i j}\right)
$$

represents lane centres, where $i$ is lane number (starting with 0 on the right) and $j$ is a subsequent point; points at the same index $j$ are linear and they lie on a line that is perpendicular to the longitudinal road axis; the array of points:

$$
\vec{e}_{i j}=\left(e_{x, i j}, e_{y, i j}, e_{z, i j}\right)
$$

defines road edges starting with the rightmost edge.

After the road geometry is defined, vehicles have to be sorted in order to provide neighbouring vehicle positions for the traffic model. A sorting algorithm is based on sorting distance, defined as maximum length from entry into the road network. Sorting distance is a continuously increasing value increasing from the input into road network and is illustrated in the Figure 8. 
a)

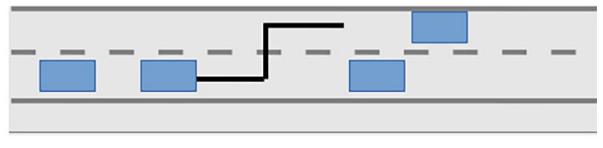

b)

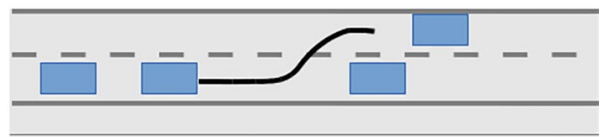

Figure 6. Continuous (a) and discrete (b) lane change

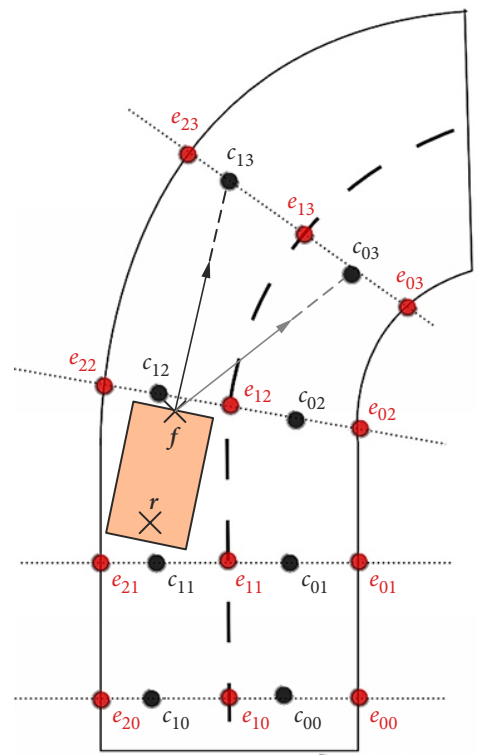

Figure 7. Definition of lane geometry

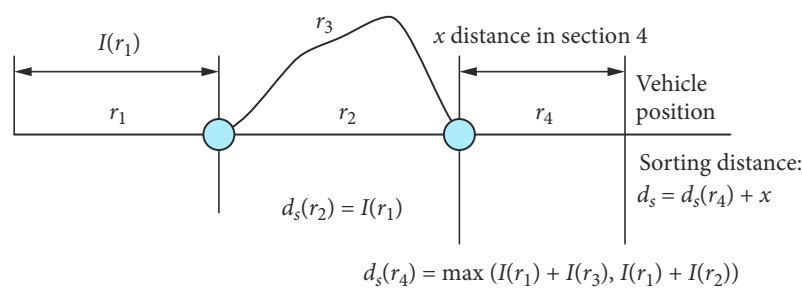

Figure 8. Definition of lane geometry

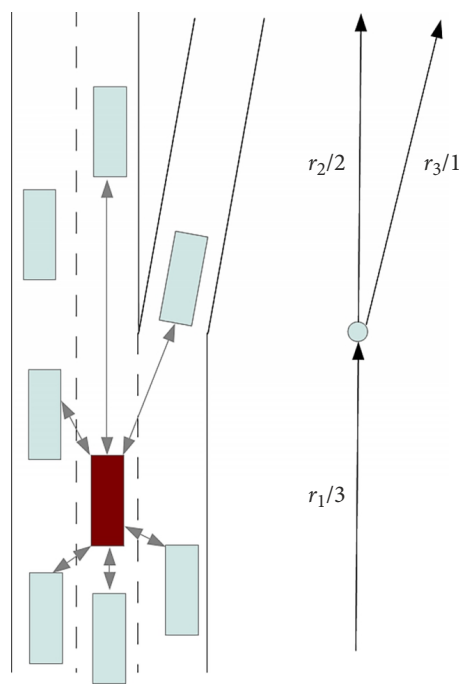

Figure 9. Adjacent vehicles and distances
After the vehicles are sorted, neighbouring vehicles on adjacent lanes can be determined. Vehicles that are of interest for lane change and vehicle following algorithms are: front-left, front, front-right and rear. Adjacent vehicles are shown in Figure 9.

\subsection{Visualization of simulation outputs}

The simulation results are post-processed and visualized in several ways:

- in operator HMI (SCADA, video-detection interface and video-surveillance 3D display);

- in instructor HMI (tunnel state, reports, time-value plots).

A video surveillance simulation module is a $3 \mathrm{D}$ computer graphics display that serves as a substitute for surveillance monitors that are being used by the tunnel operators to acquire information about situations in the tunnel. It renders states of the tunnel model in 3D space; therefore, every simulated object must have 3D coordinates and orientations defined in the simulator core itself. The visualization should be refreshed at about 20-30 frames/s in order to achieve a visually pleasant result for the observer.

Each model has its own time step, which may be longer than the maximum allowable time step for visualization and since visualization module data is synchronized at fixed time-steps, it is necessary to carry out interpolation of object movement, including translation and orientation changes between synchronization time-steps.

In Figure 10, vectors representing object positions are shown, where $x(t)$ is the position of an object in the simulator core and $y(t)$ is the visualized object. When change in speed $v(t)$ occurred at time $2 \cdot T+d t$, the movement of an object in the visualization continues along its way, but in such a way that visualized and simulated positions are synchronized by the next timestep. This is achieved by calculating object speed as:

$$
\vec{v}=\frac{\vec{x}-\vec{y}}{T} .
$$

Visualization of sensor and equipment states and control of tunnel equipment is achieved using the same SCADA interface as in a real control centre, but is connected to a computer-based simulator instead of to real tunnel controllers. The interface can be seen in Figure 11.

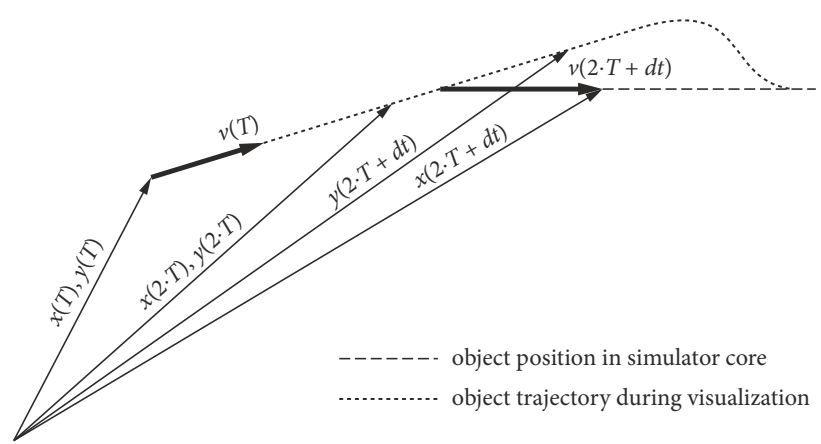

Figure 10. Interpolation of vehicle coordinates for visualization 


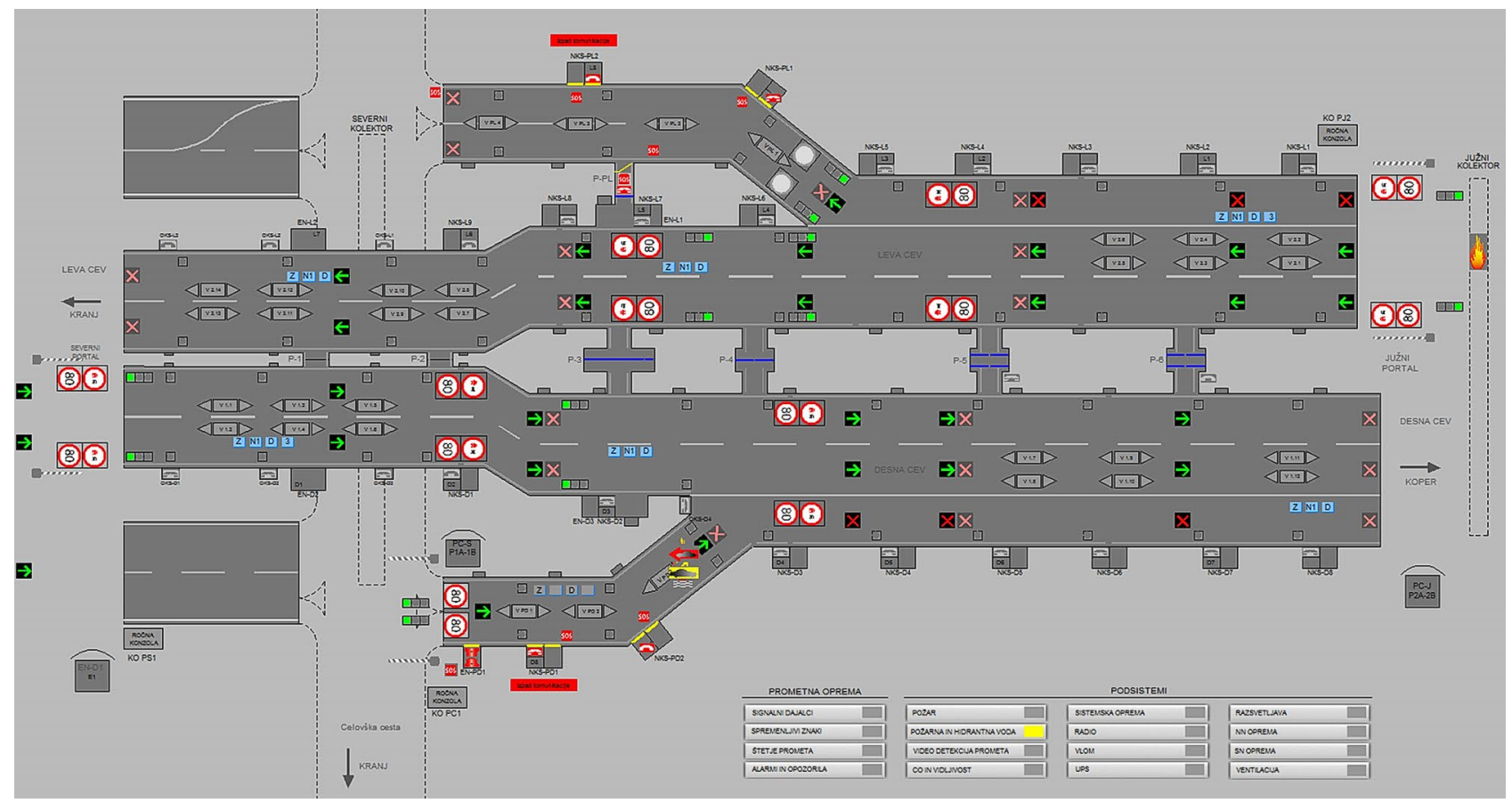

Figure 11. Operator SCADA interface

It is used to supervise and control ventilation, VMS, traffic lights, illumination, power supplies, firefighting equipment and incident detection.

The third video detection alarm interface that is used to control video surveillance is an exact replication of native interface using and showing notifications and alarms in exactly the same way. The data that is usually provided by the video processors is provided by the simulator core based on the traffic and ventilation simulation outputs:

- speed measurement, standing vehicles, static objects on the road;

- visibility, smoke concentration.

\subsection{Ventilation model tuning and validation}

The most difficult part of the unified model to be calibrated is the ventilation model. It was carried out by comparing the results with the IDA Road Tunnel Ventilation Model (Sahlin, Grozman 2003). Variables observed during the model tuning were: air velocity, air temperature, wall temperature and extinction coefficient (smoke concentration).

Air velocity calculations using the IDA model and the simulator under the same conditions show similar results that can be seen in Table.

Air temperature growth after fire can be observed in the Figure 12. Since air flow direction is from left to right and as the tunnel walls heat, the hot area increases in the direction of the air flow. Tunnel walls are the main source of cooling during the tunnel fire.

A comparison of the air temperature profile obtained with the IDA road tunnel ventilation and with a real-time simulator is slightly different for several reasons (Figure 13):

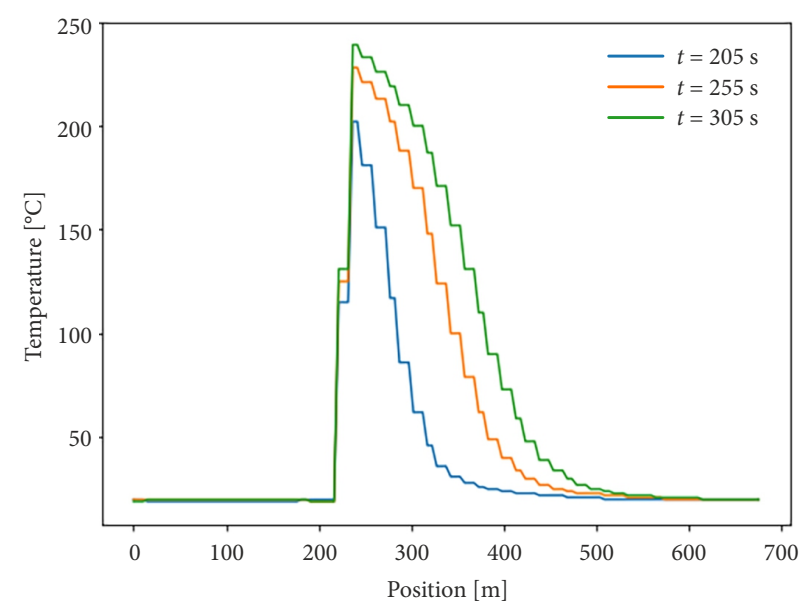

Figure 12. Air temperature profile during $50 \mathrm{MW}$ fire

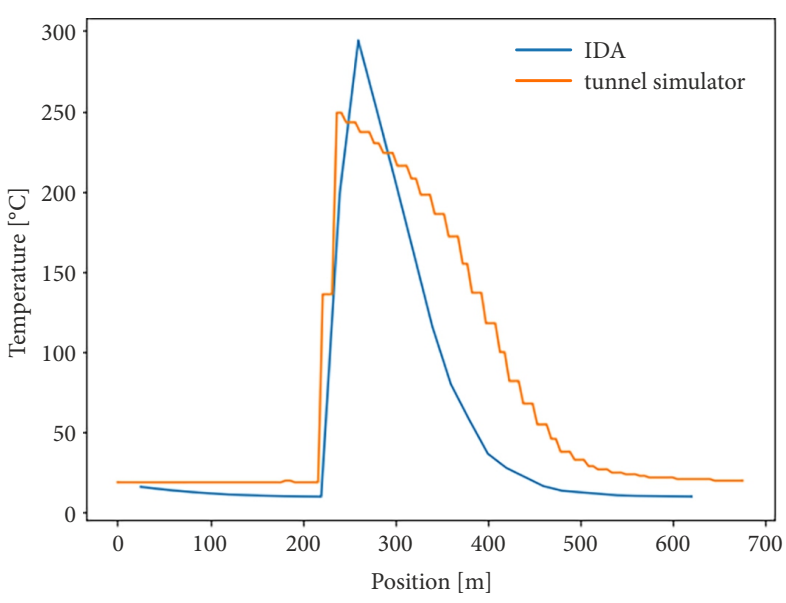

Figure 13. Air temperature compared to IDA tunnel model 
- in the real-time simulator, traffic does not stop immediately, thus influencing the air speed;

- meshing is different;

- in IDA ventilation fans are controlled at constant speed; in the tunnel simulator ventilation is controlled according to the tunnel algorithm.

Table. Comparison of simulated longitudinal air speeds during same conditions

\begin{tabular}{|l|c|c|}
\hline \multirow{2}{*}{ Tunnel section } & \multicolumn{2}{|c|}{ Longitudinal air speed [m/s] } \\
\cline { 2 - 3 } & IDA model & Simulator \\
\hline 3-lane tube & 5.9 & 5.94 \\
\hline 2-lane tube & 5.5 & 5.32 \\
\hline exit ramp & 4.6 & 4.69 \\
\hline
\end{tabular}

\section{Applications of the model}

\subsection{Operator training simulation}

The purpose of a simulator is to replace the operator workplace in a control centre with a simulated workplace, providing exactly the same user interface. Therefore, original SCADA interfaces that are being used for operational tunnels were used and connected to the simulator instead of to a real tunnel control network. A view of a simulated and a real workplace can be seen in Figure 14. The only difference is that video surveillance is replaced with a $3 \mathrm{D}$ rendering of the situation in the tunnel while maintaining exactly the same camera control interface that supports zooming and rotating.

Operators are supervised by the instructors through a special interface during training. The instructor user

a)

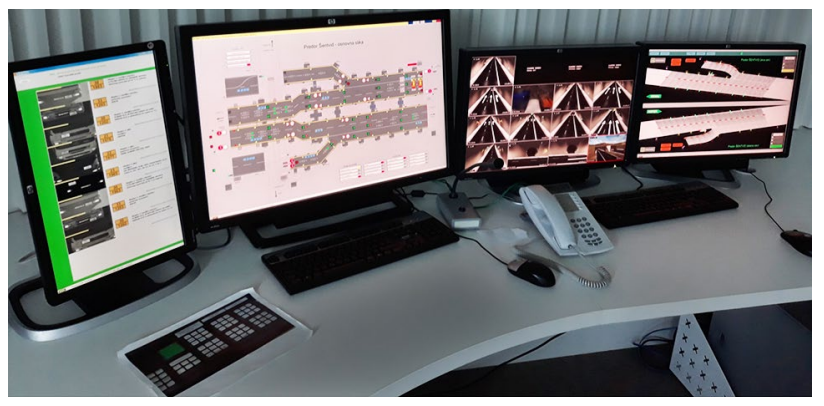

b)

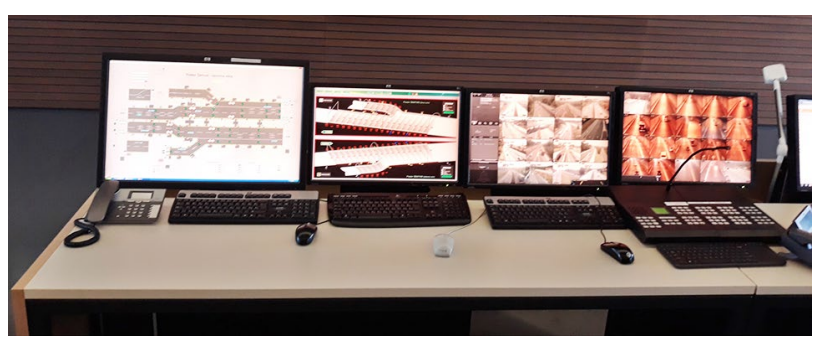

Figure 14. Operator workplace (a) and simulator (b) interface has the capability to control the simulation variables, define scenarios and supervise the situation in the tunnel during the training. The instructor is able to adjust weather conditions, traffic density, cause traffic incidents or disable systems. In the end the instructor is supposed to determine if the operator's actions were satisfactory.

Assessment of simulation outputs should answer the question of how effective the operator responses were and if the rules and procedures are being followed. This can be evaluated by observing compliance with regulations by the instructors or how many road users survive and how many are subjected to danger by observing variables such as road user and vehicle positions, vehicle speeds, air temperature and smoke concentrations. To avoid this problem, a parameter an Immediate Danger for Life and Health (IDLH) area is observed throughout the simulation. In Figure 15, vehicle positions and the IDLH area are shown over $450 \mathrm{~s}$. The vehicles at given distance are shown as colour dots, depending how many vehicles at given distance are there in parallel. After about $60 \mathrm{~s}$ the traffic is completely stopped and vehicle position points are at constant positions, forming horizontal lines. Few vehicles are affected by the fire, since most of them stopped in time. If air flow was not in the direction of the traffic, the IDLH area would be covering stopped vehicles, thus resulting in more damage. Therefore, the conditions in the Figure 15 show good response of the tunnel control system.

During the simulator development, the exact geometry of the tunnel was defined with cameras at the same positions as in the real tunnel, so the operators could observe the events through familiar views.

The operators are able to learn how to read important parameters in the tunnel and study the interaction of different systems such as ventilation control, traffic and environmental conditions. The interactions between the systems are complex and can result in unsuccessful emergency services intervention or loss of lives if not managed properly.

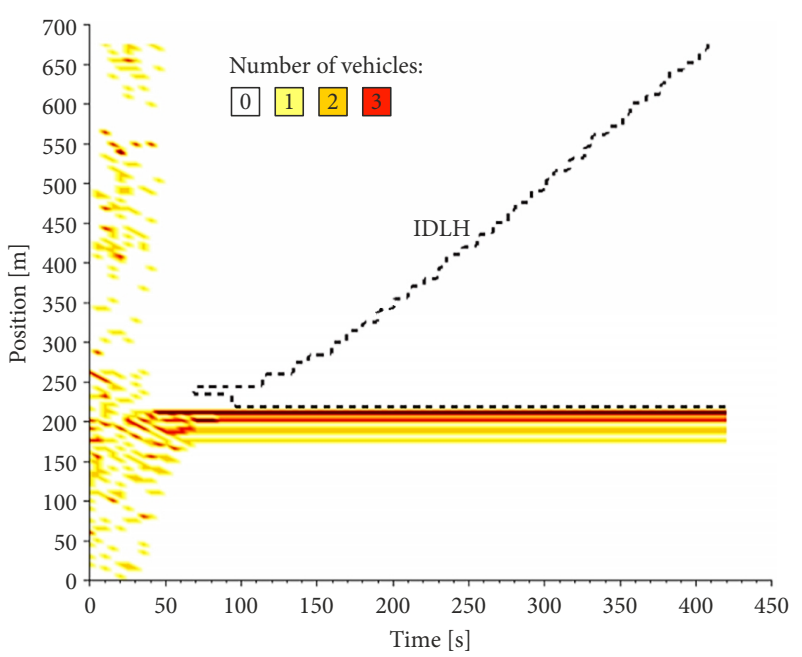

Figure 15. IDLH area during fire simulation scenario 


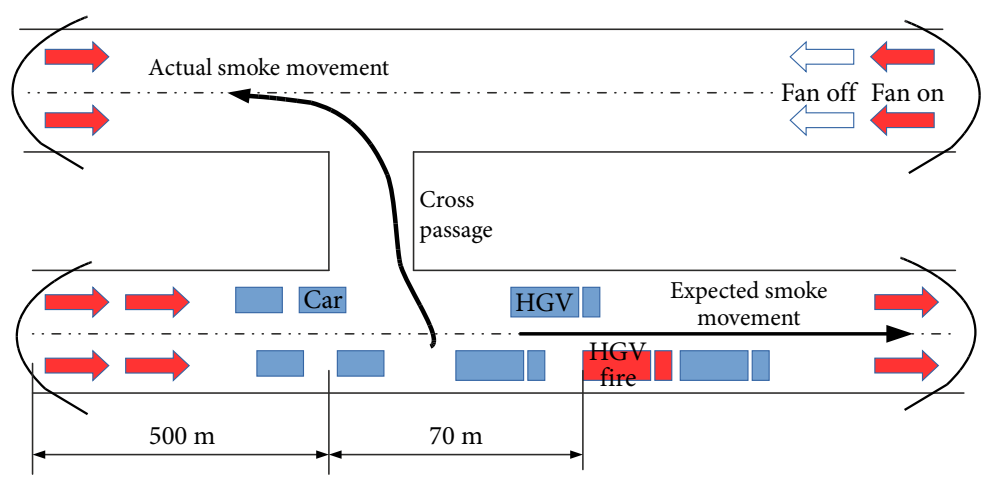

Figure 16. Vehicle and positions and fan operation during the incident

\subsection{Case study: tunnel control optimization}

An example of tunnel control optimization using the integrated tunnel model can be derived from the study of the Trojane tunnel fire accident that occurred in Slovenia in 2010 as it exposed some weaknesses of the installed tunnel control system (Luin et al. 2011). The Trojane tunnel is a twin-tube motorway tunnel that is situated on the Slovenian A1 motorway, which is part of trans-European road network and represents an important east-west link on the 5-th corridor. The road is subject to heavy traffic with yearly daily average of 37000 vehicles, among which $29 \%$ are cargo vehicles.

The incident started after a Heavy Goods Vehicle (HGV) broke down and stopped inside the tunnel. The incident detection system recorded the incident and automatically lowered speed limit and closed down the incident lane. According to the data that was obtained from the video surveillance system, especially the truck drivers maintained too short headway distance and drove at too high speed. Consequently, several vehicles collided, which resulted in an HGV fire that occurred $570 \mathrm{~m}$ from the entry portal. The evacuation of the tunnel users was meant to carry out through the emergency cross passage and the sidewalk of the opposite-direction tube, but during the incident the ventilation system did not assure smoke-free evacuation path due to unknown reason at a time, although it was discovered that everything operated according to the design procedure. The smoke that was supposed to be extracted longitudinally, in the direction of the traffic where no tunnel users remained, entered the cross passage and moved into the opposite tube. The situation is shown in the Figure 16.

To identify the cause of unexpected spreading of smoke and to improve the tunnel ventilation control and incident management, the integrated tunnel model with the simulator was used. In order to replicate the conditions, it was necessary to define vehicle positions as they were observed from the video recordings. As it is shown in the Figure 16, 4 heavy vehicles formed a jam right after the cross passage, which resulted in pressure increase in the incident tube. After simulating the fire event, following relative air pressure that is shown in the Figure 17 was obtained.

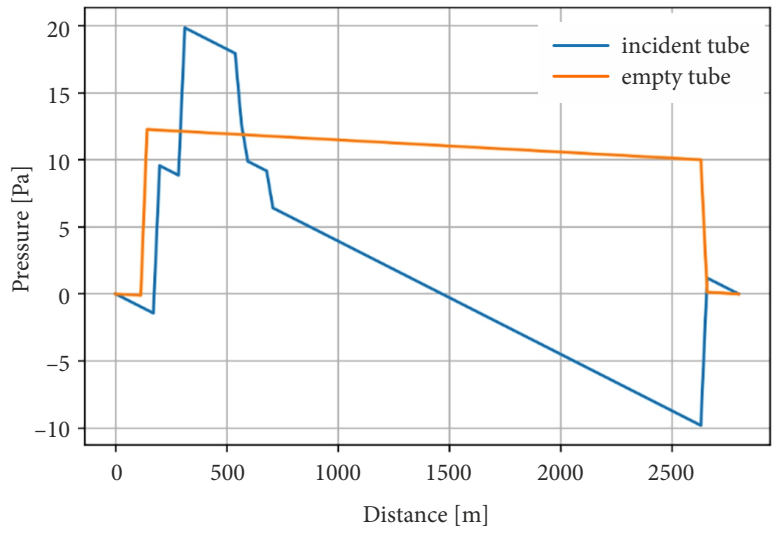

Figure 17. Pressure distribution during the incident

It is obvious that at the distance of $500 \mathrm{~m}$ from the entry portal, the pressure in the incident tube was higher than in the empty tube although according to the design strategy it was supposed to assure that the empty tube is pressurized relative to the incident tube. Apparently, the collided heavy vehicles and 4 ventilation fans at the beginning of the tunnel yielded pressure difference of about 6 $\mathrm{Pa}$, which resulted in smoke movement towards the empty tube.

It was verified if higher pressure was predominantly due to the standing vehicles and therefore the simulation was repeated under assumption of the empty tunnel tube. The results are shown in the Figure 18, where the ventilation system still causes similar conditions, except that the pressure difference drops to $4 \mathrm{~Pa}$. This means that even in an empty tunnel the designed emergency ventilation control system does not function adequately if the first cross passage is used.

To achieve sufficient pressure difference in case a fire occurs in the first $1000 \mathrm{~m}$ of the tunnel, the solution was to switch on only a single pair of fans at the tunnel entrance thus obtaining conditions in the Figure 19. This way about $2 \mathrm{~Pa}$ higher pressure is obtained in the empty tube even though there are heavy vehicles stopped after the cross passage.

The described example shows that the conditions in the tunnel are influenced by a complex set of variables defined by the traffic, ventilation, meteorological conditions 


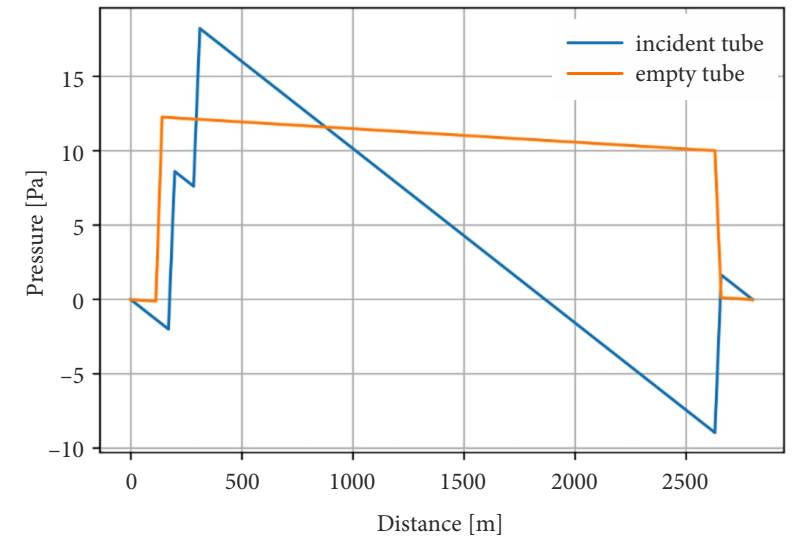

Figure 18. Pressure distribution during the incident

and tunnel equipment operation. The integrated model of the road tunnel traffic, ventilation and evacuation provides possibility to study complex scenarios that may occur in the tunnels and improve and optimize control algorithms so they can provide adequate evacuation possibilities during normal operation and during emergency conditions.

\section{Conclusions}

The simulator that is based on a real-time tunnel model provides a novel tool for evaluation of new equipment and HMI in road tunnels and also for more efficient and comprehensive road tunnel operator training. The simulator training enables the operators to become familiar with the user interface they will encounter at their workplace. However, the most important benefit of simulator training is increasing preparedness for emergency situations that have the potential to result in a large number of fatalities and material damage. Operators can train critical scenarios on simulators and practice actions that should be taken in case of emergency. This way they gain significant experience they will need in case a major accident occurs and they are the first to take action and properly inform emergency services.

Apart from road tunnel operator training, the model can be used for validation and optimization of tunnel control procedures that should be carried out during the tunnel design. It was shown on a case study of a real accident that the integrated tunnel model is a useful tool to identify causes of tunnel control malfunction and to obtain solutions to the tunnel control problems.

Key distinction of the presented approach from other modelling attempts found in the reviewed literature is the integration of different models and the simplification of the traffic, ventilation and evacuation models in order to build a unified model that is computationally undemanding and can be executed in real time or faster. This way it is possible to evaluate large sets of different scenarios for optimization and risk analysis purposes.

A merged model has some drawbacks, such as that the ventilation model is very simplified and is mostly accu-

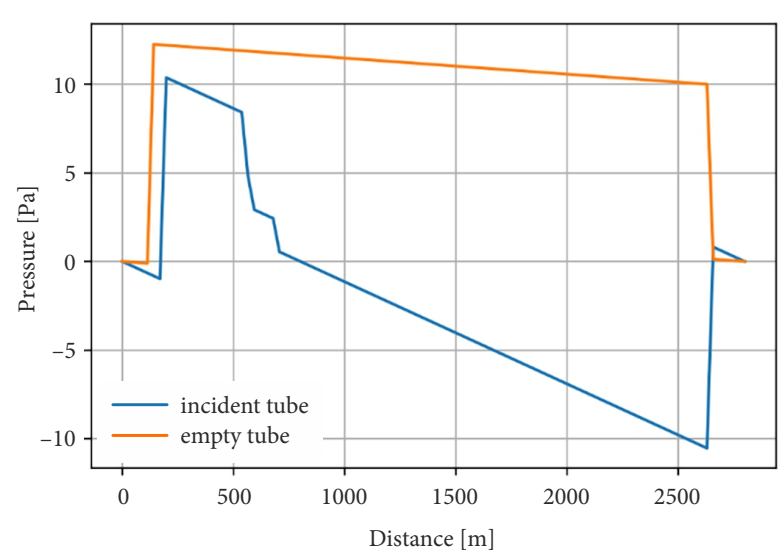

Figure 19. Pressure differences between the tubes after optimization of the algorithm

rate in 1D. If a true 3D CFD model was used, simulation would be more realistic, but it would be extremely difficult to achieve real time operation, especially merged with traffic and evacuation models that would imply constantly changing geometry and boundary conditions.

In the future, the model could be improved to be used as a risk analysis tool by executing multiple simulation runs in order to estimate damage to the tunnel and fatalities among tunnel users under different scenarios. An attempt for an improved, 3D ventilation model would also be useful in order to improve accuracy as the computer hardware is quickly developing and Graphic Processing Units (GPU) nowadays provide a cost-efficient possibility for parallel computations.

\section{Acknowledgements}

We are expressing gratitude to Urban Džindžinovič for collaboration during $3 \mathrm{D}$ visualization development and Savin Gorup from company "ASIST avtomatizacija sistemov d.o.o." (Slovenia) for detailed data on tunnel control system communication.

\section{Funding}

This work was supported by the ARRS under Grant L2-2324; and DARS, d.d. under Grant 29/20010.

\section{Author contributions}

Blaž Luin developed and tuned the unified model and performed adaptation for communication with real tunnel human-machine interfaces.

Stojan Petelin took care of project leadership and supervised the implementation and testing.

\section{Disclosure statement}

The authors have no competing financial, professional, or personal interests from other parties. 


\section{References}

Alvear, D.; Abreu, O.; Cuesta, A.; Alonso, V. 2013. Decision support system for emergency management: road tunnels, Tunnelling and Underground Space Technology 34: 13-21. https://doi.org/10.1016/j.tust.2012.10.005

Caliendo, C.; Ciambelli, P.; De Guglielmo, M. L.; Meo, M. G.; Russo, P. 2012a. Numerical simulation of different HGV fire scenarios in curved bi-directional road tunnels and safety evaluation, Tunnelling and Underground Space Technology 31: 33-50. https://doi.org/10.1016/j.tust.2012.04.004

Caliendo, C.; Ciambelli, P.; De Guglielmo, M. L.; Meo, M. G.; Russo, P. 2012b. Simulation of people evacuation in the event of a road tunnel fire, Procedia - Social and Behavioral Sciences 53: 178-188. https://doi.org/10.1016/j.sbspro.2012.09.871

Capote, J. A.; Alvear, D.; Abreu, O.; Cuesta, A.; Alonso, V. 2013. A real-time stochastic evacuation model for road tunnels, Safety Science 52: 73-80. https://doi.org/10.1016/j.ssci.2012.02.006

Carvel, R.; Rein, G.; Torero, J. L. 2009. Ventilation and suppression systems in road tunnels: some issues regarding their appropriate use in a fire emergency, in 2nd International Tunnel Safety Forum for Road and Rail, 20-22 April 2009, Lyon, France, 375-382.

Cassini, P.; Hall, R.; Pons, P. 2003. Transport of Dangerous Goods through Road Tunnels Quantitative Risk Assessment Model. Version 3.60. Reference Manual. OECD/PIARC/EU (CD-ROM).

Cassini, P. 1998. Road transportation of dangerous goods: quantitative risk assessment and route comparison, Journal of Hazardous Materials 61(1-3): 133-138.

https://doi.org/10.1016/S0304-3894(98)00117-4

Cha, M.; Han, S.; Lee, J.; Choi, B. 2012. A virtual reality based fire training simulator integrated with fire dynamics data, Fire Safety Journal 50: 12-24.

https://doi.org/10.1016/j.firesaf.2012.01.004

EC. 2004. Directive 2004/54/EC of the European Parliament and of the Council of 29 April 2004 on Minimum Safety Requirements for Tunnels in the Trans-European Road Network. Available from Internet: http://data.europa.eu/eli/dir/2004/54/oj

Fritzsche, H.-T. 1994. A model for traffic simulation, Traffic Engineering \& Control 35(5): 317-321.

Gandit, M.; Kouabenan, D. R.; Caroly, S. 2009. Road-tunnel fires: risk perception and management strategies among users, Safety Science 47(1): 105-114. https://doi.org/10.1016/j.ssci.2008.01.001

Gipps, P. G. 1981. A behavioural car-following model for computer simulation, Transportation Research Part B: Methodological 15(2): 105-111.

https://doi.org/10.1016/0191-2615(81)90037-0

Haghighat, F; Li, Y.; Megri, A. C. 2001. Development and validation of a zonal model - POMA, Building and Environment 36(9): 1039-1047.

https://doi.org/10.1016/S0360-1323(00)00073-1

Hashemkhani Zolfani, S. H.; Esfahani, M. H.; Bitarafan, M.; Zavadskas, E. K.; Arefi, S. L. 2013. Developing a new hybrid MCDM method for selection of the optimal alternative of mechanical longitudinal ventilation of tunnel pollutants during automobile accidents, Transport 28(1): 89-96. https://doi.org/10.3846/16484142.2013.782567

IEC 62381:2012. Automation Systems in the Process Industry Factory Acceptance Test (FAT), Site Acceptance Test (SAT), and Site Integration Test (SIT).
IEC TS 62603-1:2014. Industrial Process Control Systems - Guideline for Evaluating Process Control Systems - Part 1: Specifications.

Kesting, A.; Treiber, M.; Helbing, D. 2010. Enhanced intelligent driver model to access the impact of driving strategies on traffic capacity, Philosophical Transactions of the Royal Society A: Mathematical, Physical and Engineering Sciences 368: 4585-4605. https://doi.org/10.1098/rsta.2010.0084

Kesting, A.; Treiber, M.; Helbing, D. 2007. General lane-changing model MOBIL for car-following models, Transportation Research Record: Journal of the Transportation Research Board 1999: 86-94. https://doi.org/10.3141/1999-10

Krajzewicz, D.; Erdmann, J.; Behrisch, M.; Bieker, L. 2012. Recent development and applications of SUMO - simulation of urban mobility, International Journal on Advances in Systems and Measurements 5(3-4): 128-138.

Luin, B.; Petelin, S.; Vidmar, P. 2011. Trojane road tunnel operation during emergency conditions, in ISEP 2011: 19th International Symposium on Electronics in Transport, 28-29 March 2011, Ljubljana, Slovenia, 1-4.

Peacock, R. D.; Forney, G. P.; Reneke, P. A. 2013. CFAST - Consolidated Model of Fire Growth and Smoke Transport (Version 6): Technical Reference Guide. US Department of Commerce, Washington, DC, US. 129 p. https://doi.org/10.6028/NIST. SP.1026r 1

Petelin, S.; Luin, B.; Vidmar, P. 2010. Risk analysis methodology for road tunnels and alternative routes, Strojniški vestnik Journal of Mechanical Engineering 56(1): 41-51.

Sahlin, P. 1996. Modelling and Simulation Methods for Modular Continuous Systems in Buildings. Royal Institute of Technology, Stockholm, Sweden. 187 p.

Sahlin, P.; Grozman, P. 2003. IDA simulation environment a tool for Modelica based end-user application deployment, in Proceedings of the 3rd International Modelica Conference 2003, 3-4 November 2003, Linköping, Sweden, 105-114.

Shi, J.; Ren, A.; Chen, C. 2009. Agent-based evacuation model of large public buildings under fire conditions, Automation in Construction 18(3): 338-347.

https://doi.org/10.1016/j.autcon.2008.09.009

Suzuki, K.; Harada, K.; Tanaka, T. 2003. A multi-layer zone model for predicting fire behavior in a single room, Fire Safety Science 7: 851-862. https://doi.org/10.3801/IAFSS.FSS.7-851

Treiber, M.; Kesting, A. 2013. Traffic Flow Dynamics: Data, Models and Simulation. Springer. 503 p. https://doi.org/10.1007/978-3-642-32460-4

Voeltzel, A.; Dix, A. 2004. A comparative analysis of the Mont Blanc, Tauern and Gotthard tunnel fires, Routes/Roads 324: $18-34$.

Wan, H.; Du, Z.; Yan, Q.; Chen, X. 2018. Evaluating the effectiveness of speed reduction markings in highway tunnels, Transport 33(3): 647-656. https://doi.org/10.3846/transport.2018.1574

Wiedemann, R. 1974. Simulation des Straßenverkehrsflusses. Instituts für Verkehrswesen der Universität Karlsruhe, Deutschland. 85 S. (in German).

Xu, Z.; Lu, X. Z.; Guan, H.; Chen, C.; Ren, A. Z. 2014. A virtual reality based fire training simulator with smoke hazard assessment capacity, Advances in Engineering Software 68: 1-8. https://doi.org/10.1016/j.advengsoft.2013.10.004 